\title{
OCT Angiography Compared to Fluorescein and Indocyanine Green Angiography in Chronic Central Serous Chorioretinopathy
}

\author{
Michel M. Teussink, ${ }^{1}$ Myrte B. Breukink, ${ }^{1}$ Mark J. J. P. van Grinsven, ${ }^{2}$ Carel B. Hoyng, ${ }^{1}$ \\ B. Jeroen Klevering, ${ }^{1}$ Camiel J. F. Boon, ${ }^{3}$ Eiko K. de Jong, ${ }^{1}$ and Thomas Theelen ${ }^{1}$ \\ ${ }^{1}$ Department of Ophthalmology, Radboud University Medical Center, Nijmegen, The Netherlands \\ ${ }^{2}$ Diagnostic Image Analysis Group, Department of Radiology and Nuclear Medicine, Radboud University Medical Center, Nijmegen, \\ The Netherlands \\ ${ }^{3}$ Department of Ophthalmology, Leiden University Medical Center, Leiden, The Netherlands
}

Correspondence: Thomas Theelen, Department of Ophthalmology, Radboud University Medical Center, Philips van Leydenlaan 15, 6525 EX Nijmegen, The Netherlands; Thomas.Theelen@radboudumc.nl.

Submitted: April 20, 2015

Accepted: July 1, 2015

Citation: Teussink MM, Breukink MB, van Grinsven MJJP, et al. OCT angiography compared to fluorescein and indocyanine green angiography in chronic central serous chorioretinopathy. Invest Ophthalmol Vis Sci. 2015;56:5229-5237. DOI:10.1167/ iovs. $15-17140$
Purpose. Abnormal choroidal blood flow is considered important in the pathogenesis of chronic central serous chorioretinopathy (CSC). Optical coherence tomography (OCT) angiography can image ocular blood cell flow and could thus provide novel insights in disease mechanisms of CSC. We evaluated depth-resolved flow in chronic CSC by OCT angiography compared to fluorescein angiography (FA) and indocyanine green angiography (ICGA).

Methods. Eighteen eyes with chronic CSC, and six healthy controls, were included. Two human observers annotated areas of staining, hypofluorescence, and hotspots on FA and ICGA, and areas of abnormal flow on OCT angiography. Interobserver agreement in annotating OCT angiography and FA/ICGA was measured by Jaccard indices (JIs). We assessed colocation of flow abnormalities and subretinal fluid visible on OCT, and the distance between hotspots on ICGA from flow abnormalities.

REsults. Abnormal areas were most frequently annotated in late-phase ICGA and choriocapillary OCT angiography, with moderately high (median JI, 0.74) and moderate (median JI, 0.52) interobserver agreement, respectively. Abnormalities on late-phase ICGA and FA colocated with those on OCT angiography. Aberrant choriocapillary OCT angiography presented as foci of reduced flow surrounded by hyperperfused areas. Hotspots on ICGA were located near hypoperfused spots on OCT angiography (mean distance, $168 \mu \mathrm{m}$ ). Areas with current or former subretinal fluid were colocated with flow abnormalities.

Concuusions. On OCT angiography, chronic CSC showed irregular choriocapillary flow patterns, corresponding to ICGA abnormalities. These results suggest focal choriocapillary ischemia with surrounding hyperperfusion that may lead to subretinal fluid leakage.

Keywords: OCT angiography, split-spectrum amplitude decorrelation angiography, SSADA, chronic central serous chorioretinopathy, choriocapillary flow, optical coherence tomography

\begin{abstract}
Central serous chorioretinopathy (CSC) is characterized by a serous retinal detachment, sometimes accompanied by a detachment of the retinal pigment epithelium (RPE). ${ }^{1-4}$ The leakage of fluid through the RPE often occurs at the macula, resulting in central vision loss, disturbed color vision, (para-) central scotoma, metamorphopsia, and/or micropsia. ${ }^{2,4-6} \mathrm{~Pa}-$ tients with CSC are typically male (male to female ratio, 6:1), with an average age of 45 to 51 years. ${ }^{1,3}$ Besides the male sex, the most consistent risk factor appears to be the use of corticosteroids, as well as circumstances where corticosteroid levels are increased, such as Cushing disease and pregnancy. In addition, a type A personality and certain genetic factors may increase the risk for CSC. ${ }^{1,7,8}$ Although the precise pathophysiologic mechanism is still unclear, multimodal imaging indicates that subretinal fluid (SRF) accumulation in CSC results from a thickened, congested, hyperpermeable choroid leaking fluid through a dysfunctional RPE. ${ }^{9-12}$
\end{abstract}

Copyright 2015 The Association for Research in Vision and Ophthalmology, Inc iovs.arvojournals.org | ISSN: 1552-5783
Two main forms of CSC can be distinguished: the acute and chronic form. ${ }^{1,3,4,11}$ Patients with acute CSC usually present with sudden and marked central vision loss, caused by SRF leakage at the macula due to a focal leak in the RPE that is visible on fluorescein angiography (FA). ${ }^{1,2,13}$ The prognosis is favorable in this type and spontaneous recovery is often seen within 2 to 3 months. ${ }^{1}$ In contrast to acute CSC, chronic CSC is typically not self-limiting and SRF persists for more than 3 months. Patients with chronic CSC have more diffuse multifocal leakage on FA and indocyanine green angiography (ICGA), as well as irregularly distributed widespread RPE changes associated with various degrees of low-grade, more distinct leakage on angiography: diffuse retinal pigment epitheliopathy (DRPE). Such persistent serous neuroretinal detachments may cause progressive and irreversible photoreceptor damage, resulting in a far worse visual prognosis of chronic CSC as compared to the acute form..$^{2,14,15}$ 
Previous studies ${ }^{10}$ have examined blood flow in CSC by use of FA/ICGA imaging and observed choriocapillary congestion. In an ultrastructural study of a cynomolgus monkey model of CSC, damage of choriocapillary endothelial cells underneath damaged RPE cells that were covered by fibrin platelet clots has been observed, which led to the suggestion that choriocapillary hyperpermeability occurs in CSC. ${ }^{16,17}$ Blood flow in CSC has also been examined with other optical imaging modalities. For instance, choroidal hypoperfusion has been demonstrated with laser Doppler flowmetry. ${ }^{18}$

Spectral-domain optical coherence tomography (SD-OCT) is a noninvasive, high-speed, high-resolution, three-dimensional imaging technique frequently applied in patients with CSC to evaluate SRF and photoreceptor damage. ${ }^{19}$ Spectral-domain OCT may also be used to perform angiography in a noninvasive manner. Split-spectrum amplitude decorrelation angiography (SSADA) is a clinically feasible, commercialized technique that can visualize capillary blood flow, but without determination of the flow direction. ${ }^{20}$ In contrast to dye-assisted FA and ICGA imaging, OCT angiography detects movements of blood cells, instead of serum extravasation or staining of vessel walls and tissue. Split-spectrum amplitude decorrelation angiography has been used recently in the examination of abnormal vascular structures in macular telangiectasia type $2,{ }^{21}$ and of choroidal neovascularization in age-related macular degeneration, ${ }^{22,23}$ as well as chronic CSC. ${ }^{24}$ Hence, OCT angiography could provide additional information on the blood supply in CSC, and it could therefore help to better understand the underlying pathophysiology of the disease. In our current study we compared OCT angiography in chronic CSC to established angiographic imaging modalities such as FA and ICGA.

\section{METHODS}

We studied 18 eyes of 11 consecutive patients (6 male, 5 female) with chronic CSC seen at the outpatient clinic of the Department of Ophthalmology of the Radboud University Medical Center (Nijmegen, The Netherlands) from November 2014 to January 2015. All study participants gave oral informed consent for OCT-based angiography imaging. In addition, six healthy volunteers (three male, three female) with no medical history of ocular or cardiovascular diseases and/or diabetes mellitus served as controls. Ethical approval for retrospective analysis of this observational case series was granted by the institutional review board at the Radboud University Medical Center and the study adhered to the tenets of the Declaration of Helsinki. The diagnosis of chronic CSC was based on a history with persistent fluid of at least 3 months' duration, as well as RPE damage (DRPE) due to longstanding SRF. Active chronic CSC was defined by active leakage of fluid under the neuroretina confirmed by SD-OCT and by hyperfluorescent spots on ICGA and/or at least one ill-defined leakage spot on FA. Patients were included if OCT angiography, FA, and ICGA were performed on the same day to maximize the comparability of the angiographic techniques. Patients with other ocular disorders commonly associated with serous SRF, such as choroidal neovascularization, polypoidal choroidal vasculopathy, diabetic retinopathy, retinal vascular occlusion, and myopia $>6$ diopters were not eligible.

For SD-OCT, fundus autofluorescence (AF), FA and ICGA imaging, we used the Spectralis HRA + OCT device (Heidelberg Engineering, Heidelberg, Germany). Simultaneous FA and ICGA imaging were performed after patients were given a $2.5-\mathrm{mL}$ intravenous injection of $25 \%$ fluorescein solution mixed with $12.5 \mathrm{mg}$ indocyanine green (ICG-Pulsion, $25 \mathrm{mg}$; Pulsion Medical Systems SE, Feldkirchen, Germany). Fluorescein angiography and accompanying ICGA images were selected from the early phase (30-60 seconds post injection) and the late phase (6-8 minutes post injection) of the dye transit. Following ICGA/FA imaging, the presence of submacular fluid deposits was evaluated by SD-OCT.

\section{OCT Angiography}

We used a newly developed SD-OCT device (OptoVue RTVue XR Avanti AngioVue; Optovue, Inc., Fremont, CA, USA) to obtain SSADA images. This instrument has an A-scan rate of 70 $\mathrm{kHz}$ and an axial resolution in tissue of $5 \mu \mathrm{m}$, using a light source with a center wavelength of $840 \mathrm{~nm}$ and a spectral bandwidth of $45 \mathrm{~nm}$. Two consecutive B-scans (M-B frames) of 304 A-scans each along the fast-scanning axis were recorded at each of the 304 positions along the slow-scanning axis. According to this protocol, horizontal priority fast transverse ( $x$-fast) scans and vertical priority fast transverse ( $y$-fast) scans were obtained consecutively to record a three-dimensional data cube. The M-B frames were analyzed for highly decorrelated pixels, which are colocated with moving blood cells in retinal and choroidal vessels. These data were used to reconstruct three-dimensional blood flow images as described previously. ${ }^{20}$ Scans with insufficient quality due to blinking (appearing as straight, black stripes), or fixation loss, were excluded. After selecting the best scans, residual axial and saccadic motion artifacts were corrected by using the contained software (ReVue; Optovue, Inc.). The OCT angiography images of four vascular layers (inner vascular plexus, deep retinal vascular plexus, outer retina, and choriocapillaris), and a full-thickness OCT angiogram including all retinal layers and the choriocapillaris, were then exported. Examples of these images in a healthy control subject (Figs. 1A-D) and a patient with chronic CSC (Figs. 1E-H) are depicted in Figure 1.

\section{Image Readings and Annotations}

The OCT angiography images and FA/ICGA images were aligned by using a semiautomatic registration tool, developed in MeVisLab (version 2.5a; MeVis Medical Solutions AG, Bremen, Germany), that translates, rotates, and rescales the target image to accurately match three user-specified landmark locations on both the source (FA) and target (OCT angiography of the superficial layer) images. ${ }^{25}$ The same transformation matrix was applied to ICGA images for coregistration.

All image readings were performed at NANOREAD (Nijmegen Angio OCT Reading Center) by experienced and masked observers, and were done separately on the same computer. Each observer (MBB, TT) annotated areas showing leakage and hypofluorescence in the FA and ICGA images and regions in the OCT angiography images with visually evident deviations from the normal appearance. In Figure 2, the image registration and annotation processes are depicted in a flowchart together with illustrative examples. A representative example of registered images is shown in Figure 3. The OCT angiography images of five healthy volunteers, who were not part of the study, served as a reference. Images of patients and controls were anonymized and randomized, by replacing filenames with unique random numbers and subsequent sequential rearrangement according to consecutive numbering. The region-ofinterest (ROI) function of ImageJ (version 1.46r; http://imagej. nih.gov/ij/; provided in the public domain by the National Institutes of Health, Bethesda, MD, USA) was used for annotation purposes.

\section{Image Analysis}

To determine the interobserver agreement of image annotations, the number of images annotated by both observers was 

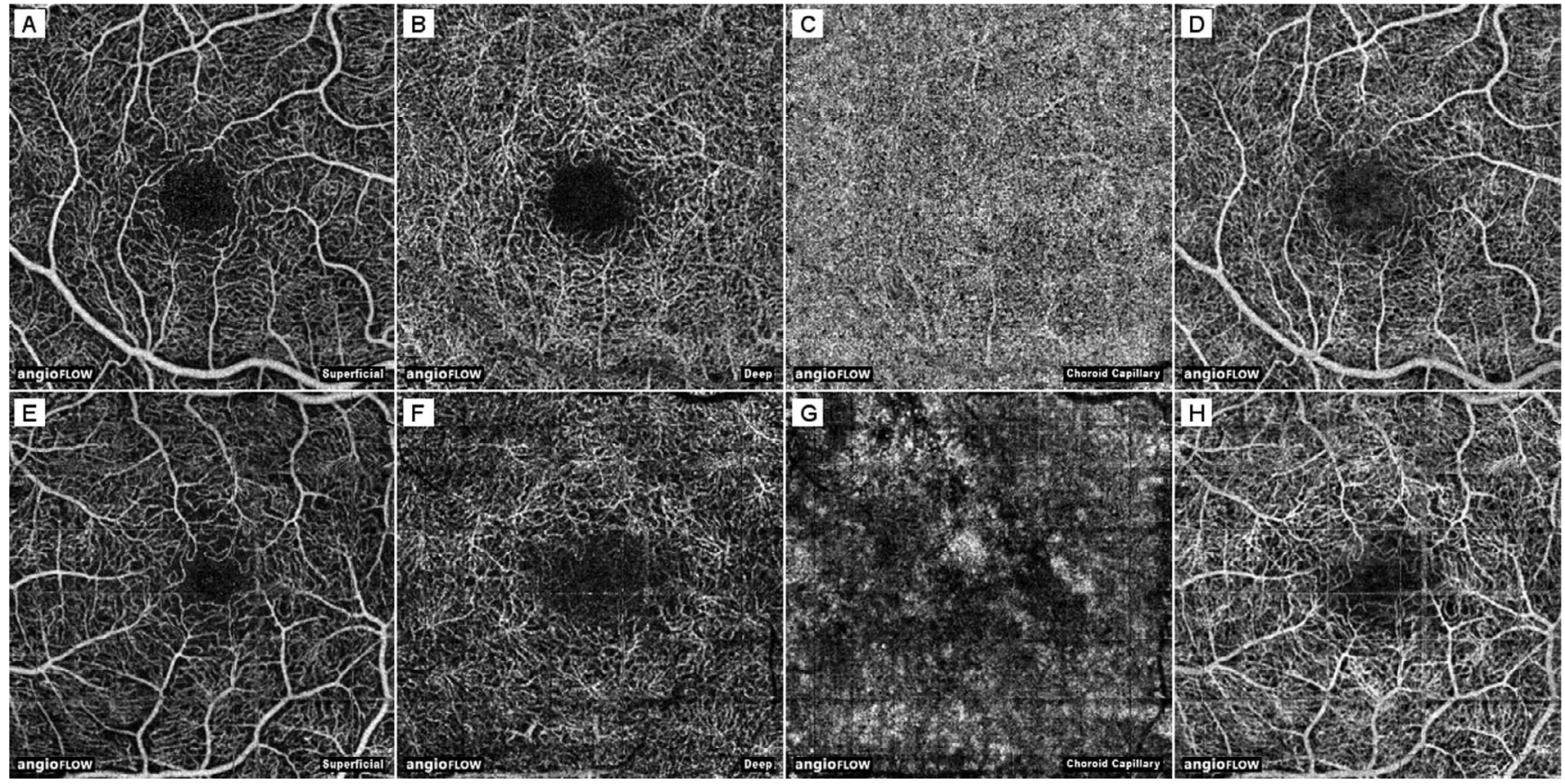

Figune 1. Optical coherence tomography angiography of a healthy subject and of a patient with chronic CSC. (A-D) Right eye of a healthy 25-yearold female. (E-H) Left eye of a patient with chronic CSC (patient 4, see Table 1). (A, E) The inner vascular plexus ("superficial"). (B, F) The deep retinal vascular plexus ("deep"). (C, G) Choriocapillaris ("choroid cap"). The choriocapillaris was defined as a 20- $\mu$ m band below the RPE-Bruch's membrane complex. It was segmented by the ReVue software and, if needed, corrected for significant segmentation errors. (D, H) Full-thickness OCT angiogram. Outer retina not shown.

determined. In case both observers annotated the image, we calculated the spatial overlap of the annotations by the Jaccard index (JI) as follows:

$$
J I\left(\mathrm{R}_{1}, \mathrm{R}_{2}\right)=\frac{\mathrm{R}_{1} \text { pixels } \cap \mathrm{R}_{2} \text { pixels }}{\mathrm{R}_{1} \text { pixels } \cup \mathrm{R}_{2} \text { pixels }} .
$$

Indices were calculated separately for each observer's set of annotations and were then pooled together. The spatial correspondence of abnormalities visible in OCT angiography images with FA/ICGA was firstly determined by the frequency of annotations on both modalities. In those cases, we calculated JIs to determine the extent of their overlap. We tested if the overlap of each combination of OCT angiography images and FA/ICGA images was different from all other combinations with the Mann-Whitney $U$ test. We also tested if this overlap, in terms of JIs, was correlated between both eyes of a patient. $P$ values $<0.05$ were considered statistically significant.

Image analysis was performed to determine the correspondence between hotspots or profound hypofluorescence in latephase ICGA images, and focal areas of decreased flow in the choriocapillary lamina on OCT angiography images, in terms of the distance between their centers. These centers were determined by the center of mass of the annotated areas. In case adjacent foci appeared confluent on ICGA images, they were often amalgamated in a single ROI as "beads on a string." A watershed algorithm was applied to split these ROIs to facilitate the distinction of different foci. Subsequently, the shortest distances between leakage hotspots or hypofluorescent foci in ICGA images and dark spots in OCT angiography images with a visually evident correspondence were calculated (Fig. 4).

\section{Fundus Autofluorescence}

To determine whether the results of OCT angiography of the choriocapillaris were affected by light-absorbing chromophores of the RPE, we compared OCT angiography images to
$\mathrm{AF}$ images that were recorded on the same day. The $\mathrm{AF}$ images were registered to OCT angiography images as described earlier. We considered OCT angiography signals above or below the mean \pm 2 standard deviations of apparently uninvolved areas as abnormal choriocapillary flow. Uninvolved areas were determined by a lack of annotations, absence of large vessel shadows, and in the near vicinity of the image border, as irregular flow at the image borders was common even in healthy subjects. Thresholded pixels of increased or decreased flow were overlaid on the registered AF images. Finally, two observers (MMT, TT) evaluated whether there was any colocation of focally changed AF signals and choriocapillary flow.

\section{RESUlts}

Patient demographics are depicted in Table 1.

\section{Interobserver Agreement}

A total of 255 images were analyzed, including 100 FA and ICGA images of patients, and 125 and 30 OCT angiography images of patients and healthy subjects, respectively. In 132 images ( $52 \%$ ), abnormalities were annotated by both observers (83 FA/ICGA images and 49 OCT angiography images). An example of image annotations by both observers is shown in Figure 5. The corresponding JIs as indicators of interobserver agreement and numbers of annotated images are summarized in Table 2. The FA and ICGA images were annotated with moderate agreement (median JIs, 0.43-0.67). Of the various OCT angiography images, choriocapillary flow images were most frequently annotated by both observers (80\% of the patient images, $17 \%$ of the control images) with moderate interobserver agreement (median JI, 0.52). The OCT angiography images of retinal layers were annotated less often than choriocapillary flow images. The interobserver agreement in 


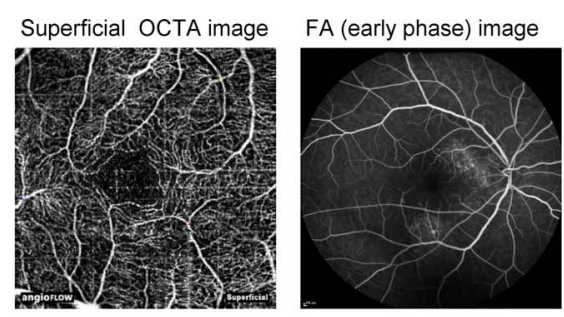

Checkered overlay mage
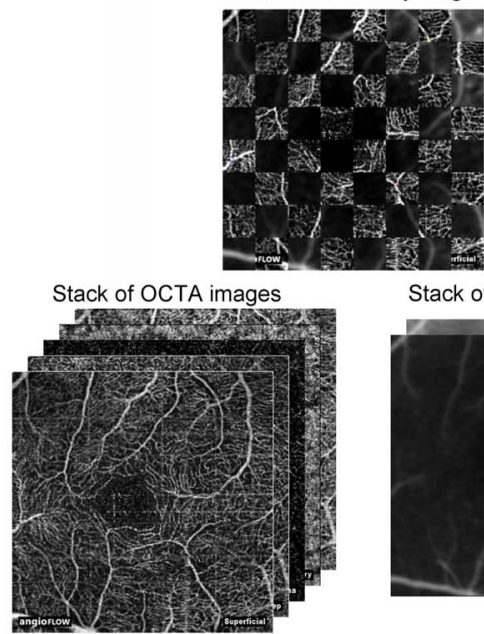

Stack of $F A / I C G A$ images
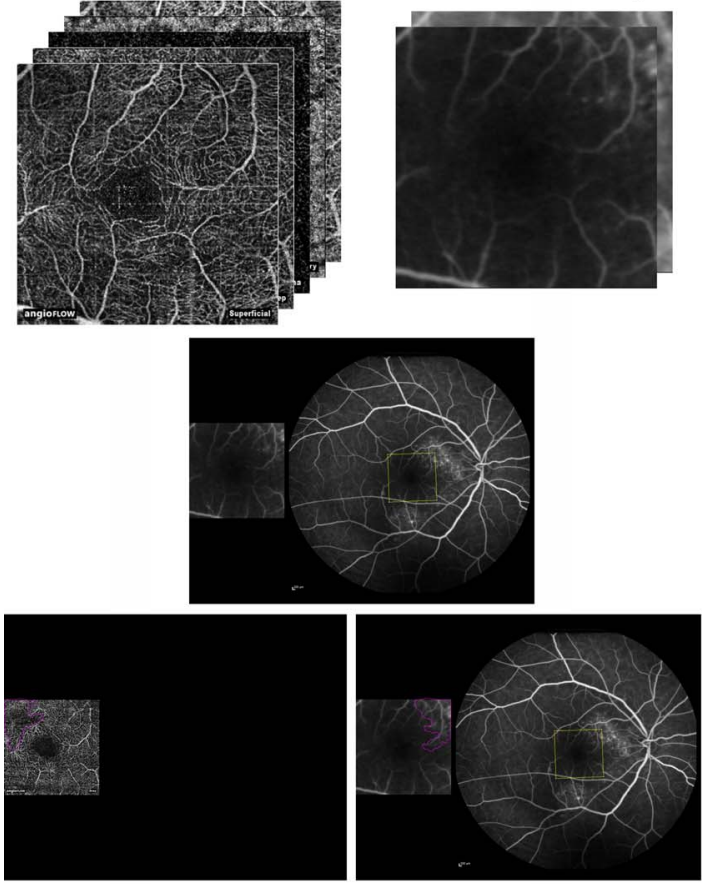

Original images

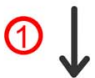

Determine the needed transformation with different image overlay options:

- Checkerboard

- Semi-transparancy of one image

- Matching of image landmarks

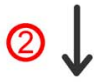

Identical transformation of the ICGA image of the same stack. Transformations of OCTA images are not needed.

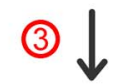

Final display image

(4) $\downarrow$

\begin{tabular}{l}
\hline Annotations by human graders \\
(5) $\downarrow$ \\
Calculate overlap of annotations \\
with Jaccard's index
\end{tabular}

FIGURE 2. Flowchart of the image registration and annotation procedures. Original images, that is, superficial retinal OCT angiography and FA images, were registered by an observer, using a variety of image overlay options (step 1). Simultaneously acquired ICGA images were transformed identically to the FA image (step 2). The final display image, with a standard layout, was then created, which included the original FA/ICGA image containing contextual information (step 3). Annotations were made by human observers (step 4), after which the overlap of annotations was calculated by Jaccard's index (step 5). See text for details.

annotating abnormal choriocapillary flow was similar to FA and ICGA, both in terms of the frequency of annotations and the agreement scores.

\section{General Correspondence Between OCT Angiography and FA/ICGA}

Table 3 summarizes the overlap of annotated abnormal areas observed with various angiographic imaging modalities expressed in JIs. Choriocapillary flow images and late-phase FA/ ICGA images corresponded best in determining abnormality with moderately low overlap of the annotated areas (median JI of both FA and ICGA versus choriocapillary, 0.41). In general, there was a poor overlap between annotated changes on FA and OCT angiography images, with median JIs ranging from 0 to 0.05 . The correspondence between aberrant choriocapillary

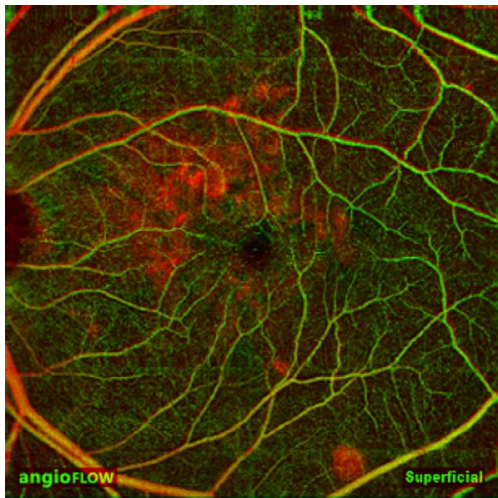

Figure 3. Example of image registration. Overlay of an early-phase FA image (red) registered to a superficial OCT angiography image (green), with overlapping vessels indicated in yellow. 

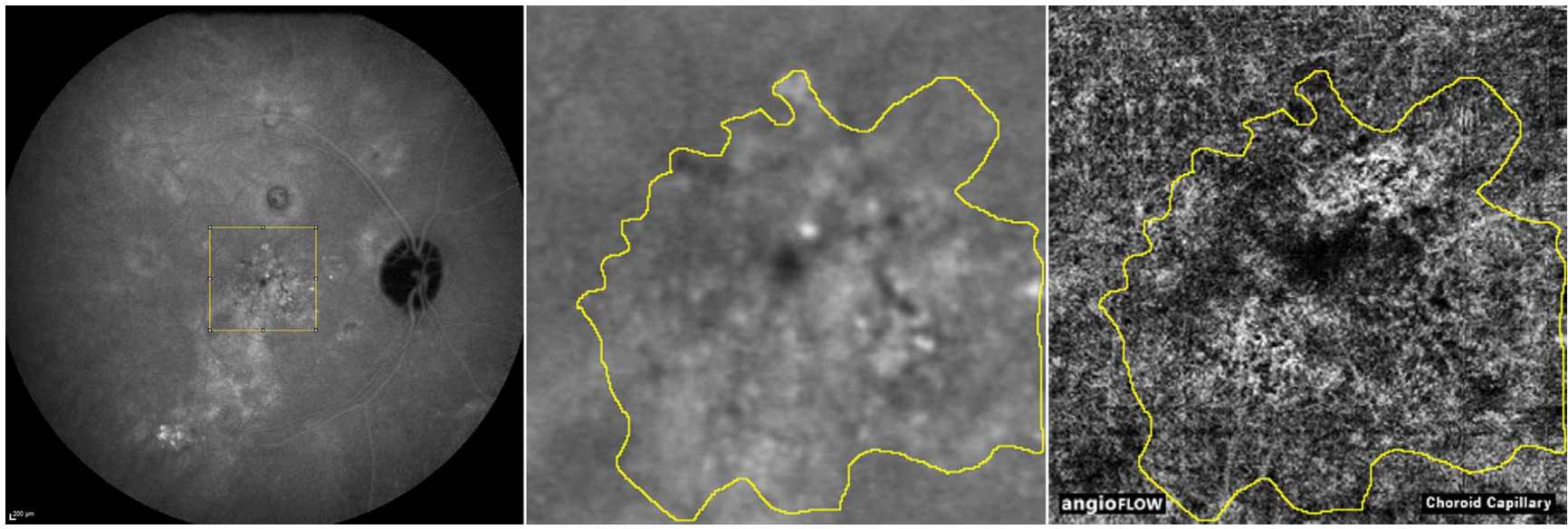

FIGURE 4. Annotation and quantitative analysis of hyperfluorescent areas on ICGA and aberrant flow on OCT angiography. Left image: Late-phase ICGA image of a patient with chronic CSC with location of the registered image (yellow frame). Middle image: Detail of abnormalities in the registered ICGA image. Right image: The aberrant flow pattern on OCT angiography of the choriocapillaris is colocated with the annotated region on late-phase ICGA; the darkest region corresponds with a hypofluorescent spot on ICGA, bordered by increased flow colocated to the leakage hotspot and late staining. The annotated region is depicted by the yellow line.

flow and abnormalities on late-phase FA/ICGA was higher than the correspondence of abnormalities in early or late FA images and abnormalities in retinal OCT angiography images $(P<$ 0.001). In general, the JIs showed a significant correlation between both eyes (Spearman's $\rho=0.59, P=4.5 \cdot 10^{-7}$ ).

The annotated choriocapillary flow patterns had a highly variable appearance. In some cases, we observed mottled flow patterns with patches of increased and decreased flow. Mostly, however, we observed irregular patterns with large and semiconfluent patches of decreased flow adjacent to similar patches with increased flow. A small number of patches with decreased choriocapillary flow corresponded with RPE detachments visible on SD-OCT. Several other patches corresponded with focal areas of a thickened RPE below an SRF pocket, which appeared to produce shadow artifacts on latephase ICGA. In general, light-absorbing fluorophores in the $\mathrm{RPE}$, as visualized by AF imaging, had no apparent correspondence with the appearance of choriocapillary flow on OCT angiography of CSC. A few sporadic hyper-AF areas corresponded with decreased flow areas, although not all hyper-AF in the same images corresponded with decreased flow and vice-versa. The same was true for the correspondence between hypo-AF and increased flow, and for other combinations. Areas of aberrant choriocapillary flow were generally colocated with locations of current SRF accumulation visible on SD-OCT, or with locations where SRF had resolved. The aberrant flow patterns appeared similarly heterogeneous in treatment-naive patients and treated subjects, with no consistent differences in eyes that received micropulse laser treatment or photodynamic therapy.

\section{Correspondence Between Hotspots on ICGA and Aberrant Choriocapillary Flow}

We also studied the relationship between decreased choriocapillary flow and hotspots or hypofluorescence on ICGA (Fig.

TABLE 1. Clinical Characteristics of the Patients Included in This Study

\begin{tabular}{|c|c|c|c|c|}
\hline D/Sex/Age, y & $\begin{array}{c}\text { Eye(s) } \\
\text { Included }\end{array}$ & $\begin{array}{l}\text { Signs of Chronic } \\
\text { CSC on FA, } \\
\text { ICGA, and OCT }\end{array}$ & $\begin{array}{l}\text { Active Chronic } \\
\text { CSC on FA, } \\
\text { ICGA, and OCT }\end{array}$ & $\begin{array}{c}\text { Previous } \\
\text { Treatment(s) }\end{array}$ \\
\hline $1 / \mathrm{F} / 55$ & OD & Yes & Yes & $1 \times \mathrm{MP}$ \\
\hline $2 / \mathrm{M} / 58$ & OD & Yes & Yes & NA \\
\hline \multirow[t]{2}{*}{$3 / \mathrm{F} / 64$} & OD & Yes & Yes & NA \\
\hline & OS & Yes & Yes & NA \\
\hline \multirow[t]{2}{*}{$4 / \mathrm{M} / 40$} & OD & Yes & No & NA \\
\hline & Os & Yes & Yes & NA \\
\hline 5/M/71 & OD & Yes & No & $2 \times$ PDT \\
\hline $6 / F / 56$ & OD & Yes & Yes & NA \\
\hline \multirow[t]{2}{*}{$7 / F / 38$} & OD & Yes & No & NA \\
\hline & OS & Yes & Yes & $1 \times \mathrm{PDT}$ \\
\hline \multirow[t]{2}{*}{$8 / F / 56$} & OD & Yes & No & $1 \times \mathrm{MP}$ \\
\hline & OS & Yes & Yes & NA \\
\hline \multirow[t]{2}{*}{$9 / \mathrm{M} / 59$} & OD & Yes & Yes & $2 \times \mathrm{MP}, 1 \times \mathrm{PDT}$ \\
\hline & OS & No & No & NA \\
\hline \multirow[t]{2}{*}{$10 / \mathrm{M} / 62$} & OD & No & No & NA \\
\hline & OS & Yes & Yes & NA \\
\hline \multirow{2}{*}{$11 / \mathrm{M} / 47$} & OD & Yes & Yes & NA \\
\hline & OS & Yes & No & $2 \times \mathrm{MP}, 1 \times \mathrm{PDT}$ \\
\hline
\end{tabular}

F, female; M, male; MP, micropulse laser therapy; NA, not applicable; OD, right eye; OS, left eye; PDT, half-dose photodynamic therapy. 

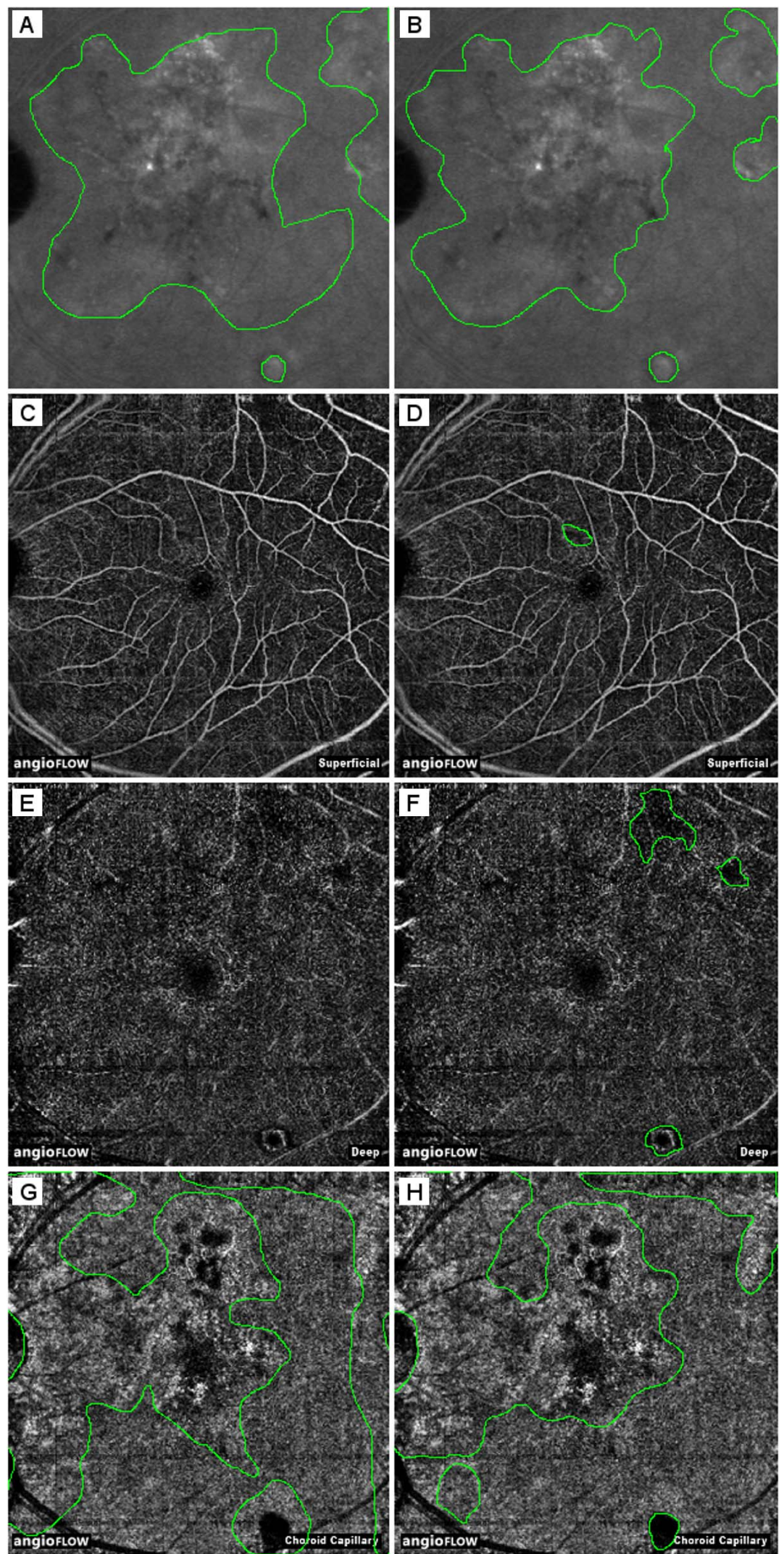

Figure 5. Examples of image annotations. (A, B) Late-phase ICGA images (left eye of patient $8,6 \times 6 \mathrm{~mm}$ ). (C, D) Superficial OCT angiography images. (E, F) Deep OCT angiography images. (G, H) Choriocapillary OCT angiography images. Left column: Annotations by observer 1 . Right column: Annotations by observer 2. The JIs of overlap between late-phase ICGA and superficial- and deep-OCT angiography were 0.006 and 0.022 , respectively (observer 2). The JIs of overlap of late-phase ICGA with choriocapillary OCT angiography were 0.43 and 0.55 for observers 1 and 2 , respectively. 
TABLE 2. Interobserver Agreement of Annotated Vascular Abnormalities in FA, ICGA, and OCT Angiography Images

\begin{tabular}{|c|c|c|c|c|c|}
\hline & \multirow{2}{*}{$\begin{array}{c}\text { JI } \\
\text { Mean (SD) }\end{array}$} & \multirow[b]{2}{*}{ Median } & \multicolumn{3}{|c|}{$\begin{array}{c}\text { Annotations Made } \\
\text { by Observers }\end{array}$} \\
\hline & & & Both & Either & None \\
\hline \multicolumn{6}{|l|}{ FA/ICGA } \\
\hline FA, early phase & $0.46(0.29)$ & 0.35 & 19 & 1 & 5 \\
\hline FA, late phase & $0.59(0.23)$ & 0.58 & 20 & 2 & 3 \\
\hline ICGA, early phase & $0.43(0.29)$ & 0.47 & 22 & 1 & 2 \\
\hline ICGA, late phase & $0.67(0.15)$ & 0.74 & 22 & 2 & 1 \\
\hline \multicolumn{6}{|l|}{ OCT angiography } \\
\hline Superficial & $0.28(0.22)$ & 0.28 & 4 & 9 & 18 \\
\hline Deep & $0.27(0.19)$ & 0.26 & 11 & 10 & 10 \\
\hline Outer retina & $0.47(0.17)$ & 0.40 & 7 & 16 & 8 \\
\hline Choroid cap & $0.49(0.22)$ & 0.52 & 21 & 5 & 5 \\
\hline FT-OCTA & $0.5(0.2)$ & 0.53 & 6 & 11 & 14 \\
\hline
\end{tabular}

Choroid cap, choriocapillaris; FT-OCTA, full-thickness OCT angiogram; SD, standard deviation.

4). In most areas with decreased flow on OCT angiography we were unable to observe a corresponding, well-defined focus of abnormal fluorescence on late-phase ICGA (75 of 117 areas, or 64.1\%). The remaining areas with decreased flow, however, overlapped largely with hypofuorescent areas on ICGA. Twelve (29.3\%) of 41 leakage areas and hotspots on late-phase ICGA appeared to be connected with areas of decreased choriocapillary flow on OCT angiography, versus 24 (42.9\%) of 56 hypofluorescent spots on late-phase ICGA. The mean distance between hotspots on ICGA and matched areas with decreased signal in choriocapillary flow images on OCT angiography was $168 \mu \mathrm{m}(\mathrm{SD}, 133 \mu \mathrm{m})$, or 17 pixels. The mean distance of hypofluorescent spots was $133 \mu \mathrm{m}$ (SD, $70 \mu \mathrm{m}$ ), or 13 pixels. Areas of increased flow, however, were often found adjacent to leakage hotspots on ICGA. For choriocapillary flow images and corresponding FA/ICGA and AF images of all included patients and controls, see Supplementary Figure S1.

\section{Discussion}

In this study, we compared OCT angiography characteristics of patients with chronic CSC to FA and ICGA findings. We found typical changes in the choriocapillary flow pattern of chronic CSC patients, which corresponded to areas affected by SRF accumulation. There was moderate interobserver agreement on changes in choriocapillary flow images of CSC patients, and there was moderate spatial correspondence between late FA/ ICGA and choriocapillary flow images.

Kitaya et al. ${ }^{18}$ have used laser Doppler flowmetry to show that eyes with CSC have reduced choroidal blood flow relative to the unaffected fellow eye. Using combined FA/ICGA imaging, Scheider et al. ${ }^{26}$ also have confirmed the reduction in choroidal blood flow in CSC and demonstrated that the hypoperfusion persists after clinical improvement. In our study, changes of the choriocapillary flow pattern on OCT angiography appeared as focally increased and decreased pixel values, indicating coexisting increased and decreased flow, respectively. Even though the appearance of these changes was significantly different from the fairly homogeneous appearance of the healthy choriocapillary layer, there was only a moderate interobserver agreement. Obviously, the border between the appearance of healthy and affected tissue in OCT angiography may not easily be drawn between observers. This phenomenon has been observed earlier in a study that evaluated the
TABLE 3. Overlap of Image Annotations on FA/ICGA and OCT Angiography in 11 Patients With Chronic CSC

\begin{tabular}{llcl}
\hline \multicolumn{1}{c}{ FA/ ICGA } & $\begin{array}{c}\text { OCT } \\
\text { Angiography }\end{array}$ & Mean JI (SD) & Median \\
\hline FA, early phase & Superficial & $0.04(0.1)$ & 0 \\
& Deep & $0.06(0.1)$ & 0.01 \\
& Choroid cap & $0.25(0.21)$ & 0.22 \\
& FT-OCTA & $0.1(0.19)$ & 0 \\
FA, late phase & Superficial & $0.03(0.06)$ & 0 \\
& Deep & $0.09(0.14)$ & 0.02 \\
& Choroid cap & $0.38(0.22)$ & 0.41 \\
& FT-OCTA & $0.11(0.15)$ & 0.05 \\
ICGA, early phase & Superficial & $0.12(0.2)$ & 0 \\
& Deep & $0.09(0.12)$ & 0.04 \\
& Choroid cap & $0.27(0.18)$ & 0.24 \\
ICGA, late phase & FT-OCTA & $0.1(0.15)$ & 0.05 \\
& Superficial & $0.04(0.06)$ & 0 \\
& Deep & $0.08(0.11)$ & 0.05 \\
& Choroid cap & $0.41(0.19)$ & 0.41 \\
& FT-OCTA & $0.14(0.2)$ & 0.05 \\
\hline
\end{tabular}

agreement among highly experienced retinal specialists in grading of FA images for the presence of active choroidal neovascularization. ${ }^{27} \mathrm{~A}$ multimodal approach in angiographic techniques may help to improve the identification of pathologic changes. One has to keep in mind, however, that OCT angiography measures blood flow dependent on intravascular cell movements, while FA/ICGA images the flow of blood plasma, independent of cell movement. Therefore, these three techniques give blood flow information that is supplementary to each other.

On OCT angiography, we observed choriocapillary hypoperfusion with hyperperfusion in the surrounding area. This is consistent with results of earlier studies, showing focal filling defects in the choriocapillaris with dilated and tortuous feeding arterioles and dilated venules. ${ }^{10,28-30}$ These filling defects may be caused by capillary congestion and/or rarefaction. ${ }^{10}$ Areas of irregular choriocapillary texture on OCT angiography partially colocated to areas with late staining on ICGA. Also, many hotspots on late-phase ICGA, if present, were in close proximity of relative ischemia on OCT angiography within those areas of irregular texture. Taken together, these data suggest that reduced blood perfusion in the choriocapillaris may be surrounded by reactive hyperperfusion. Hyperperfusion leads to increased hydrostatic pressure within the fenestrated choriocapillaris. Together with chronic hypoxic damage, this may lead to disintegrity of the continuity of the RPE ${ }^{31}$ and to SRF leakage with serous neuroretinal detachment in chronic CSC. ${ }^{32,33}$

According to our data, the abnormal vascular situation in the choriocapillary layer appeared to persist even after resolution of SRF and seemed to be independent of any kind of treatment. It is still unclear why different treatment modalities may lead to successful fluid resorption. The mechanism of action of photodynamic therapy has been postulated to include short-term choriocapillary hypoperfusion and long-term choroidal vascular remodeling with subsequent reduction of vascular hyperpermeability and leakage. ${ }^{19,34,35}$ On the short term, the hypoperfusion may lead to more ischemic RPE damage. Because of the long period between photodynamic therapy and our study measurements, we may have missed any therapeutic effects visible on OCT angiography. It may be useful to prospectively follow up patients after photodynamic therapy by OCT angiography to learn more about the therapeutic mechanism of this treatment. Subthreshold micropulse diode laser treatment is applied in an attempt 
to trigger the regeneration of the RPE and a long-term metabolic increase at the chorioretinal junction, ${ }^{36}$ which may increase SRF resorption by the RPE. ${ }^{37}$ Micropulse laser treatment is not thought to affect the choriocapillaris directly. The lack of effects on choriocapillary flow in patients after micropulse laser treatment is therefore not surprising.

Our study was mainly limited by the small number of patients and the retrospective and cross-sectional data acquisition. As a consequence, we have studied a combination of treatment-naive patients and patients who were previously treated by micropulse laser or photodynamic therapy once or multiple times. We do not have pretreatment data and we did not monitor patients over time. To further improve our knowledge of the vascular changes in chronic CSC, prospective and longitudinal studies are required to monitor vascular changes during the natural course and during different treatment strategies. Although we found no consistent effects of chromophores in the RPE on the appearance of choriocapillary flow, save for a few sporadic cases, a comprehensive analysis of potential confounders on the appearance of OCT angiography signals was out of the scope of this study. This is important in OCT angiography studies of retinal diseases in which layers with high light-absorption are affected. Therefore, future studies should include SD-OCT and AF imaging to determine potential imaging artifacts. The fields of view on OCT angiography were of considerably smaller size than those currently used in ICGA or SD-OCT. Peripheral areas with obvious FA/ICGA irregularities were therefore missed. Future studies should aim to include the complete posterior pole with all angiographic abnormalities by OCT angiography scanning. This could be achieved by a montage of many high-resolution OCT angiography scans or by improved OCT hardwaresoftware combinations capable of visualizing larger fundus areas with high resolution.

In conclusion, OCT angiography showed significant textural changes of the choriocapillary flow pattern in chronic CSC patients. Our results suggest foci of ischemia surrounded by reactive choroidal hyperperfusion as an important factor in the pathogenesis of chronic CSC. Prospective OCT angiography studies on chronic CSC are needed to better understand the pathogenesis and treatment response of this visually threatening disease.

\section{Acknowledgments}

The device used in this study was provided by Rockmed B.V., Oirschot, The Netherlands.

Supported by grants from the Foundation Fighting Blindness, Baltimore, MD, USA, and by the Gelderse Blindenstichting, Velp, The Netherlands. The Foundation Fighting Blindness and the Gelderse Blindenstichting had no role in the design and conduct of the study; collection, management, analysis, and interpretation of the data; preparation, review, or approval of the manuscript; and decision to submit the manuscript for publication.

Disclosure: M.M. Teussink, None; M.B. Breukink, None; M.J.J.P. van Grinsven, None; C.B. Hoyng, None; B.J. Klevering, None; C.J.F. Boon, None; E.K. de Jong, None; T. Theelen, None

\section{References}

1. Liew G, Quin G, Gillies M, Fraser-Bell S. Central serous chorioretinopathy: a review of epidemiology and pathophysiology. Clin Exp Opbthalmol. 2013;41:201-214.

2. Wang M, Munch IC, Hasler PW, Prunte C, Larsen M. Central serous chorioretinopathy. Acta Ophthalmol. 2008;86:126145.
3. Nicholson B, Noble J, Forooghian F, Meyerle C. Central serous chorioretinopathy: update on pathophysiology and treatment. Surv Ophthalmol. 2013;58:103-126.

4. Gemenetzi M, De Salvo G, Lotery AJ. Central serous chorioretinopathy: an update on pathogenesis and treatment. Eye (Lond). 2010;24:1743-1756.

5. Yannuzzi LA. Central serous chorioretinopathy: a personal perspective. Am J Ophthalmol. 2010;149:361-363.

6. Maaranen TH, Tuppurainen KT, Mantyjarvi MI. Color vision defects after central serous chorioretinopathy. Retina. 2000; 20:633-637.

7. de Jong EK, Breukink MB, Schellevis RL, et al. Chronic central serous chorioretinopathy is associated with genetic variants implicated in age-related macular degeneration. Ophthalmology. 2015;122:562-570.

8. Miki A, Kondo N, Yanagisawa S, Bessho H, Honda S, Negi A. Common variants in the complement factor $\mathrm{H}$ gene confer genetic susceptibility to central serous chorioretinopathy. Ophthalmology. 2014;121:1067-1072.

9. Gass JD. Pathogenesis of disciform detachment of the neuroepithelium. Am J Ophthalmol. 1967;63(suppl):1-139.

10. Prunte C, Flammer J. Choroidal capillary and venous congestion in central serous chorioretinopathy. Am J Ophthalmol. 1996;121:26-34.

11. Spaide RF, Hall L, Haas A, et al. Indocyanine green videoangiography of older patients with central serous chorioretinopathy. Retina. 1996;16:203-213.

12. Guyer DR, Yannuzzi LA, Slakter JS, Sorenson JA, Ho A, Orlock D. Digital indocyanine green videoangiography of central serous chorioretinopathy. Arch Ophthalmol. 1994;112:10571062.

13. Eandi CM, Ober M, Iranmanesh R, Peiretti E, Yannuzzi LA. Acute central serous chorioretinopathy and fundus autofluorescence. Retina. 2005;25:989-993.

14. Loo RH, Scott IU, Flynn HW Jr, et al. Factors associated with reduced visual acuity during long-term follow-up of patients with idiopathic central serous chorioretinopathy. Retina. 2002;22:19-24.

15. Wang MS, Sander B, Larsen M. Retinal atrophy in idiopathic central serous chorioretinopathy. Am J Ophthalmol. 2002; 133:787-793.

16. Yoshioka H, Katsume Y, Akune H. Experimental central serous chorioretinopathy in monkey eyes: fluorescein angiographic findings. Ophthalmologica. 1982;185:168-178.

17. Yoshioka H, Katsume Y. Experimental central serous chorioretinopathy, III: ultrastructural findings. Jpn J Ophthalmol. 1982;26:397-409.

18. Kitaya N, Nagaoka T, Hikichi T, et al. Features of abnormal choroidal circulation in central serous chorioretinopathy. $\mathrm{BrJ}$ Ophthalmol. 2003;87:709-712.

19. Liegl R, Ulbig MW. Central serous chorioretinopathy. Ophthalmologica. 2014;232:65-76.

20. Jia Y, Tan O, Tokayer J, et al. Split-spectrum amplitudedecorrelation angiography with optical coherence tomography. Opt Express. 2012;20:4710-4725.

21. Spaide RF, Klancnik JM Jr, Cooney MJ. Retinal vascular layers in macular telangiectasia type 2 imaged by optical coherence tomographic angiography. JAMA Ophthalmol. 2015;133:6673.

22. Jia Y, Bailey ST, Wilson DJ, et al. Quantitative optical coherence tomography angiography of choroidal neovascularization in age-related macular degeneration. Ophthalmology. 2014;121: 1435-1444.

23. de Carlo TE, Bonini Filho MA, Chin AT, et al. Spectral-domain optical coherence tomography angiography of choroidal neovascularization. Ophthalmology. 2015;122:1228-1238. 
24. Bonini Filho MA, de Carlo TE, Ferrara D, et al. Association of choroidal neovascularization and central serous chorioretinopathy with optical coherence tomography angiography [published online ahead of print May 21, 2015]. JAMA Ophthalmol. doi:10.1001/jamaophthalmol.2015.1320.

25. van Grinsven MJ, Buitendijk GH, Brussee C, et al. Automatic identification of reticular pseudodrusen using multimodal retinal image analysis. Invest Ophthalmol Vis Sci. 2015;56: 633-639.

26. Scheider A, Nasemann JE, Lund OE. Fluorescein and indocyanine green angiographies of central serous choroidopathy by scanning laser ophthalmoscopy. Am J Ophthalmol. 1993; 115:50-56.

27. Holz FG, Jorzik J, Schutt F, Flach U, Unnebrink K. Agreement among ophthalmologists in evaluating fluorescein angiograms in patients with neovascular age-related macular degeneration for photodynamic therapy eligibility (FLAP-study). Ophthalmology. 2003;110:400-405.

28. Nishiyama Y, Mori K, Murayama K, Yoneya S. Quantitative analysis of indocyanine green angiographic image in central serous chorioretinopathy. Jpn J Ophthalmol. 2001;45:116.

29. Iida T, Kishi S, Hagimura N, Shimizu K. Persistent and bilateral choroidal vascular abnormalities in central serous chorioretinopathy. Retina. 1999;19:508-512.

30. Ferrara D, Mohler KJ, Waheed N, et al. En face enhanced-depth swept-source optical coherence tomography features of chronic central serous chorioretinopathy. Ophthalmology. 2014;121:719-726.

31. Uyama M, Matsunaga H, Matsubara T, Fukushima I, Takahashi $\mathrm{K}$, Nishimura T. Indocyanine green angiography and pathophysiology of multifocal posterior pigment epitheliopathy. Retina. 1999;19:12-21.

32. Hussain D, Gass JD. Idiopathic central serous chorioretinopathy. Indian J Ophthalmol. 1998;46:131-137.

33. Slakter JS, Yannuzzi LA, Guyer DR, Sorenson JA, Orlock DA. Indocyanine-green angiography. Curr Opin Ophthalmol. 1995;6:25-32.

34. Karakus SH, Basarir B, Pinarci EY, Kirandi EU, Demirok A. Long-term results of half-dose photodynamic therapy for chronic central serous chorioretinopathy with contrast sensitivity changes. Eye (Lond). 2013;27:612-620.

35. Koytak A, Erol K, Coskun E, Asik N, Ozturk H, Ozerturk Y. Fluorescein angiography-guided photodynamic therapy with half-dose verteporfin for chronic central serous chorioretinopathy. Retina. 2010;30:1698-1703.

36. Brinkmann R, Schule G, Neumann J, et al. Selective retina therapy: methods, technique, and online dosimetry [in German]. Ophthalmologe. 2006;103:839-849.

37. Klatt C, Saeger M, Oppermann T, et al. Selective retina therapy for acute central serous chorioretinopathy. Br J Ophthalmol. 2011;95:83-88. 